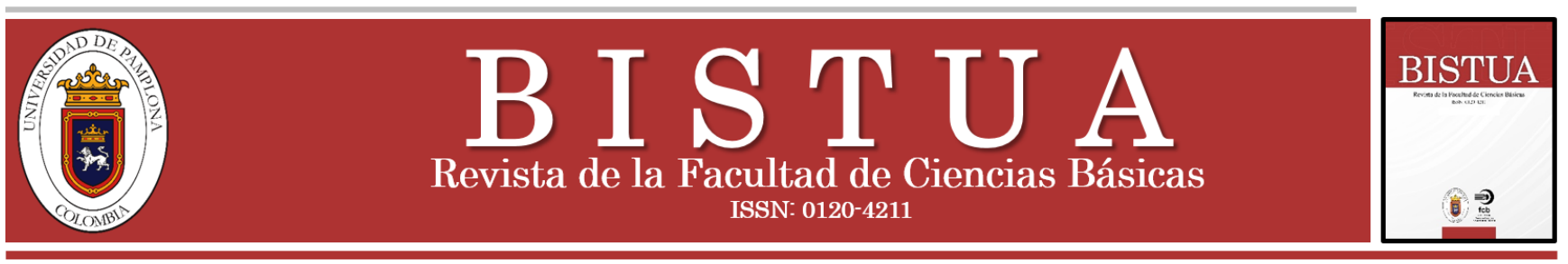

\title{
Efectos de la presión hidrostática sobre los modos defectivos en un cristal fotónico bidimensional semiconductor con geometría hexagonal
}

\section{Effects of hydrostatic pressure on defect modes in a semiconductor two-dimensional photonic crystal with hexagonal geometry}

\author{
Juan Sebastián Soto Perdomo ${ }^{a}$; Emiro Segundo Arrieta Jiménez ${ }^{b}$ \\ ${ }^{a}$ Facultad de Ciencias Exactas y Naturales, Universidad Surcolombiana, Colombia. \\ ${ }^{b}$ Facultad de Educación, Universidad Surcolombiana, Colombia. \\ Contactos: u20151134910@usco.edul.co; emiro.arrieta@usco.edu.co
}

Recibido: Enero 10, 2020. Aceptado: Abril 9, 2020

https://doi.org/10.24054/01204211.v1.n1.2020.4172

\begin{abstract}
Resumen
Las propiedades ópticas de las estructuras caracterizadas mediante una función dieléctrica periódica como los cristales fotónicos $(\mathrm{CFs})$, ha despertado interés por sus aplicaciones en diversos campos de la óptica y la optoelectrónica. Cuando la periodicidad de los CFs se rompe por la inserción de defectos, permite el confinamiento de modos de luz. En esta contribución, utilizando el método de expansión en ondas planas para el modo de polarización transversal magnética y el Software computacional $M A T L A B$, se estudia el efecto de la presión hidrostática sobre la estructura de bandas en un cristal fotónico bidimensional (CF-2D) con red hexagonal, compuesto por agujeros de aire en un fondo de material semiconductor de fosfuro de galio $(\mathrm{GaP})$, que se caracteriza por tener el índice de refracción más alto entre los semiconductores binarios y su rango de transmisión se utiliza en aplicaciones de luz visible e infrarrojo cercano. Inicialmente, se estudia la relación de dispersión del cristal fotónico regular (sin defecto) y se analiza la zona de Banda Fotónica Prohibida (BFP); posteriormente se aumenta la presión hidrostática, generando que la estructura de banda fotónica presente un desplazamiento a regiones de alta frecuencia. Posteriormente, utilizando la técnica de la supercelda se considera un defecto puntual dentro de la estructura, que reemplaza un agujero de aire por material semiconductor, que genera estados permitidos o modos de defecto en el interior del bandgap fotónico. Al aumentar la presión hidrostática se observa que el ancho del bandgap fotónico permanece inalterado y los modos de defecto presentan un desplazamiento a valores de altas frecuencias.
\end{abstract}

Palabras clave: Cristal fotónico; presión hidrostática; estructura de bandas; método de expansión en ondas planas; modos localizados.

\section{Introducción}

Los avances en la física de los semiconductores ha permitido manipular por completo las propiedades electrónicas de ciertos materiales. Análogamente, el campo de la fotónica abrió una rama novedosa de medios ópticos representados por estructuras artificiales con una modulación periódica en la constante dieléctrica, una solución al problema de control y manipulación óptico es conocido como Cristal fotónico (CF) [1]. Dependiendo de su estructura, se puede dividir en tres grandes categorías, unidimensionales (1D), bidimensionales (2D) y tridimensionales (3D) [2]. En

\section{Abstract}

The optical properties of structures characterized by a periodic dielectric function such as photonic crystals (PCs) have sparked interest in their applications in various fields of optics and optoelectronics. When the periodicity of the CFs is broken by the insertion of defects, it allows the confinement of light modes. In this contribution, using the plane wave expansion method for the transversal magnetic polarization mode and the MATLAB computational Software, the effect of hydrostatic pressure on the band structure in a two-dimensional photonic crystal with a hexagonal lattice, composed of air holes in a background of gallium phosphide $(\mathrm{GaP})$ semiconductor material, which is characterized as it has the highest refractive index among binary semiconductors and its transmission range is used in visible light and near infrared applications. Initially, the scattering ratio of the regular photonic crystal (without defect) is studied and analyzed the photonic band gap zone (PBG); then the hydrostatic pressure is increased, which causes the photonic band structure to present a displacement to regions of high frequency. Continuing this analysis, using the supercell technique, a point defect is considered within the structure that replaces an air hole with semiconductor material, which generates allowed states or defect modes inside the photonic bandgap. As the hydrostatic pressure increases, it is observed that the width of the photonic bandgap remains unchanged and the defect modes present a shift to high frequency values.

Keywords: Photonic crystal; hydrostatic pressure; band structure; plane wave expansion method; localized modes.

1987, E. Yablonovitch planteó la utilización de ciertos materiales para aumentar la eficiencia del láser, al proponer que la emisión espontánea de luz podría estar prohibida dentro de un intervalo de banda fotónica; donde la luz no puede propagarse a través de la estructura en ninguna dirección [3]. Simultáneamente, S. Jhon descubrió que una modificación en un medio periódico puede localizar la luz para un cierto rango de frecuencias, por lo tanto, la onda electromagnética incidente que cae en el intervalo de banda fotónica, será reflejada completamente por el cristal [4]; en consecuencia, no existen modos de Bloch correspondientes que puedan propagarse a través del cristal fotónico [5]. Esta 
capacidad de los cristales fotónicos para inhibir la propagación de la luz con frecuencias bien definidas tiene una gran semejanza con las propiedades electrónicas de los semiconductores [6]. Mientras, el comportamiento de los electrones en un semiconductor está descrito por la ecuación de Schrödinger, el comportamiento de la luz en un CF se describe utilizando las ecuaciones de Maxwell.

Las propiedades ópticas de las estructuras caracterizadas mediante una función dieléctrica periódica provoca una región de energías prohibidas para los fotones. Como consecuencia de la periodicidad, las ondas electromagnéticas se dispersan dentro del cristal dando lugar a la interferencia destructiva de las ondas, lo que genera una banda fotónica prohibida (BFP) [7]. La existencia de esta banda no permite la propagación de las ondas electromagnéticas a ciertas frecuencias dentro del CF.

$\mathrm{Al}$ igual que en un semiconductor, los electrones libres están sometidos a un potencial periódico que tiene la capacidad de modificarse al introducir defectos en la periodicidad de la red cristalina; en los CFs estos defectos se crean al añadir, modificar o eliminar parte de los componentes de la red, lo que permite el confinamiento o la guía de modos electromagnéticos con una alta eficiencia dentro de la zona PBG, conocidos como modos localizados y modos de propagación [8].

\subsection{Antecedentes}

Los cristales fotónicos han generado gran interés por sus aplicaciones en diversos campos de la óptica y la optoelectrónica como transistores ópticos, nanointerruptores ópticos, guías de onda, sensores de temperatura y presión, entre otros [9], debido a que se construyen a partir de diferentes tipos de materiales.

E. Yablonovitch [10] trabajo con semiconductores, A. Sanchez et al. [11] con polímeros, A. Gharaali y Z. Zare [12] con metales, O. Berman et al. [13] con superconductores y Liu et al. [14] con metamateriales, generando mayor investigación en el desarrollo de dispositivos capaces de suplir la necesidad de obtener un control más preciso de la luz.

Las regiones de BFP y los modos electromagnéticos en los CF se pueden describir con base al desarrollo de las ecuaciones de Maxwell mediante métodos computacionales. M. Lin y R. Jao [15] trabajaron el método de elementos finitos (FEM), A. Taflove y S. Hagness [16] el método de diferencias finitas en el dominio del tiempo (FDTD), J. Shumpert [17] el método de momentos, M. korobogatiy y J. Yang [18] el método de la matriz de trasferencia y K. Sakoda, H. Sozuer et al. [19-20] el método de expansión en ondas planas. Esta investigación se centra en estudiar el comportamiento de la estructura de banda fotónica al someter un CF bajo un agente externo que altere las propiedades ópticas de los materiales. Aly et al. [21] investigaron los efectos que el campo magnético tiene sobre la permitividad de los metales que componen un CF-2D, utilizando el MEOP, analizaron como la EBF se puede modificar y controlar fuertemente ajustando el campo magnético externo. C. Duque y M. Mora [22] consideraron los efectos del campo magnético, la temperatura y la presión hidrostática que tienen sobre la EBF de un CF-2D semiconductor, manifestando como el aumento de la presión hace que la BFP se desplace a regiones de alta frecuencia y la temperatura genere un pequeño desplazamiento a regiones de bajas frecuencias. $\mathrm{N}$. Porras y C. Duque [23] utilizaron el MEOP para analizar la dependencia de la presión hidrostática y la temperatura sobre la EBF de un CF bidimensional, para la polarización TM y TE, reportaron que al modificar la presión hidrostática y la temperatura generaba desplazamientos de la EBF, además generaba cambios en el ancho de las bandas prohibidas superiores para la polarización TM y para la polarización TE permanecían inalteradas. F. Segovia y H. Vinck [24] trabajaron la EBF de un CF-2D defectivo con red cuadrada compuesto por cilindros infinitos de un semiconductor en un fondo de aire utilizando el MEOP, analizaron los efectos de la temperatura y la presión hidrostática, sus resultados presentaron un desplazamiento del modo defectivo a altas frecuencias a medida que aumentaban la presión hidrostática manteniendo una temperatura constante dentro de la BFP. Las anteriores investigaciones se centraron en estudiar estructuras compuestas por GaAs, de acuerdo con estos resultados, surge la necesidad de un estudio adicional sobre sobre un material semiconductor perteneciente también al grupo III-V que no altere drásticamente el valor de la permitividad dieléctrica al variar un agente externo.

Este trabajo, por lo tanto, presenta un estudio de la influencia de la presión hidrostática sobre el comportamiento de la EBF para analizar la región de BFP en un CF-2D regular y defectivo con geometría hexagonal, compuesto por agujeros de aire en un fondo de material de fosfuro de galio (GaP), utilizando el método de expansión en ondas planas para el modo de polarización TM y la técnica de la supercelda. El documento está organizado de la siguiente manera: en la sección 2, se presenta las ecuaciones generales para calcular la EBF del CF-2D. La sección 3 y 4 presenta los resultados y el análisis numérico de las simulaciones respectivamente. En la sección 5 se presentan las conclusiones del estudio.

\section{Metodología}

La estructura de banda fotónica se puede determinar estudiando la dinámica del campo electromagnético a partir de las ecuaciones de Maxwell en medios materiales lineales, isotrópicos, inhomogéneos y no magnéticos [25].

$$
\frac{1}{\varepsilon(\vec{r})} \nabla \times[\nabla \times \vec{E}(\vec{r})]=\frac{\omega^{2}}{c^{2}} \vec{E}(\vec{r})
$$




$$
\nabla \times\left[\frac{1}{\varepsilon(\vec{r})} \nabla \times \vec{H}(\vec{r})\right]=\frac{\omega^{2}}{c^{2}} \vec{H}(\vec{r})
$$

donde $c$ es la velocidad de la luz y $\omega$ es la frecuencia de la luz. En el plano $x y$ la constante dieléctrica $\varepsilon(\vec{r})$ es una función periódica de $\vec{r}$, que satisface la condición $\varepsilon(\vec{r})=$ $\varepsilon(\vec{r}+\vec{R})$, siendo $\vec{R}=l \overrightarrow{a_{1}}+m \overrightarrow{a_{2}}$ el vector de translación de la red espacial real, $\overrightarrow{a_{1}}$ y $\overrightarrow{a_{2}}$ son los vectores de red primitivos y $l$ y $m$ son números enteros.

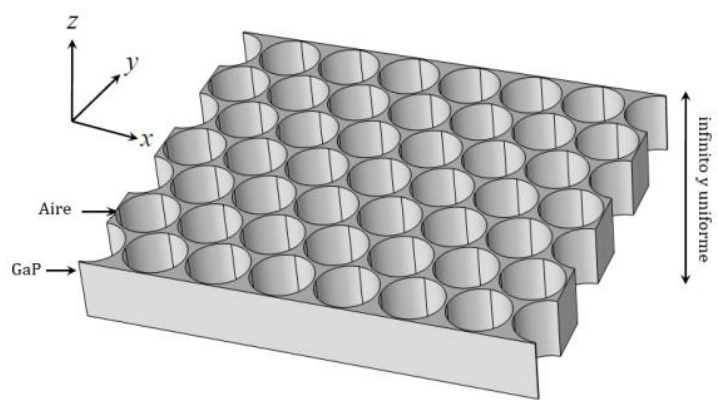

Figura 1. Esquema del CF-2D de red hexagonal compuesto por agujeros de aire insertados en $\mathrm{GaP}$ de radio $\mathrm{R}=0.48 a$.

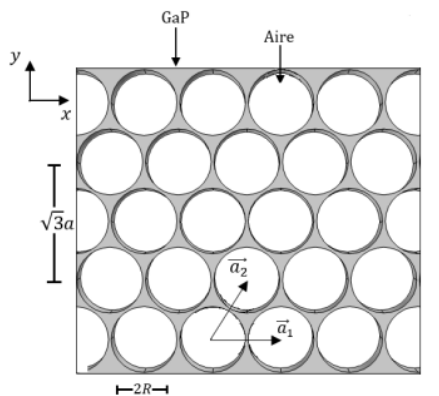

(a)

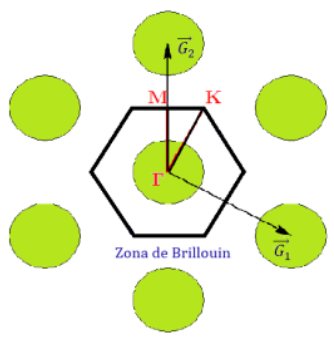

(b)
Figura 2. (a) Estructura del cristal fotónico en el espacio real. (b) espacio recíproco y primera zona de Brillouin.

De igual forma que, en los sistemas semiconductores para la red cristalina, las componentes de los vectores de la red recíproca para una red hexagonal están definidos como:

$$
\begin{gathered}
\vec{G}_{1}=\frac{4 \pi}{a \sqrt{3}}\left(\frac{\sqrt{3}}{2} \hat{x}-\frac{1}{2} \hat{y}\right) \\
\vec{G}_{2}=\frac{4 \pi}{a \sqrt{3}} \hat{y}
\end{gathered}
$$

Para el tipo de polarización TM, el campo magnético está confinado en el plano $x y$ y el campo eléctrico es perpendicular al plano, es decir: $\left(H_{x}, H_{y}, 0\right) ;\left(0,0, E_{z}\right)$.

A partir de la Ec.(1) para los modos TM y usando el teorema de Bloch-Floquet [26], la componente $\vec{E}(\vec{r})$ se expresa como

$$
\vec{E}(\vec{r})=\hat{z} E_{\vec{k}, n}(\vec{r}) e^{i \vec{k} \cdot \vec{r}}
$$

donde $E_{\vec{k}, n}$ es una función periódica. Los subíndices indican que la función periódica depende del vector de onda $\vec{k}$ y de $\operatorname{los} n$ estados propios del modo electromagnético.

Utilizando el análisis de Fourier, se considera que las ondas electromagnéticas que se propagan son ondas planas, por lo tanto, el campo eléctrico y la función dieléctrica se puede expandir en términos de sumas discretas:

$$
\begin{aligned}
& \vec{E}_{\vec{k}, n}=\hat{z} \sum_{G} E_{G}^{T M} e^{i(\vec{k}+\vec{G}) \cdot \vec{r}} \\
& \frac{1}{\varepsilon(\vec{r})}=\sum_{G} \chi_{G} e^{i \vec{G} \cdot \vec{r}}
\end{aligned}
$$

donde $E_{G}^{T M}$ y $\chi_{G}$ son los coeficientes de expansión de Fourier para $E_{\vec{k}, n} \mathrm{y} \frac{1}{\varepsilon(\vec{r})}$ respectivamente. Sustituyendo las Ec.(6) y Ec.(7) en la Ec.(1), obtenemos una ecuación de valores propios.

$$
\sum_{G} \chi_{G^{\prime}-G}\left[\left(G_{x}+k_{x}\right)^{2}+\left(G_{y}+k_{y}\right)^{2}\right] E_{G}^{T M}=\frac{\omega^{2}}{c^{2}} E_{G^{\prime}}^{T M}
$$

La Ec.(8) se conoce como la ecuación maestra y define un sistema infinito de ecuaciones para $E_{G}^{T M}$, con valores propios $\omega^{2} / c^{2}$. Solucionando esta ecuación, se puede calcular la estructura de bandas de los modos electromagnéticos dentro del cristal fotónico 2D. Para que el problema tenga solución numérica, es necesario truncar la expansión en series de Fourier del campo eléctrico y de la función dieléctrica a un subconjunto finito, diagonalizando las matrices para cada valor de $G$.

En esta investigación se considera el CF-2D con red hexagonal compuesto por agujeros de aire insertados periódicamente en el material semiconductor de GaP, como se muestra en la Fig.1, la permitividad dieléctrica del GaP como función de la presión hidrostática y la temperatura, está definida como [27]:

$$
\varepsilon_{G a P}(\mathrm{P}, \mathrm{T})=\varepsilon_{a} e^{\alpha_{1} T+\alpha_{2} P}
$$

para $T<200 \mathrm{~K}$, se considera $\varepsilon_{a}=10.86, \quad \alpha_{1}=5.4 \times$ $10^{-5} \mathrm{~K}^{-1}$ y $\alpha_{2}=-0.97 \times 10^{-3} \mathrm{kbar}^{-1}$. Para nuestro estudio el material semiconductor se encuentra a una temperatura de $24 \mathrm{~K}$ y se toman valores de presión de 0, 40, 80 y $100 \mathrm{kbr}$. Los coeficientes de expansión de Fourier relacionados con la permitividad dieléctrica se definen como:

$$
\chi_{G}=\frac{1}{S_{u}} \int \frac{1}{\varepsilon(\vec{r})} e^{-i \vec{G} \cdot \vec{r}} d \vec{r}
$$

donde la integración se realiza dentro del área de la celda unitaria del cristal $S_{u}$. Los coeficientes de expansión en series de Fourier de la permitividad dieléctrica se expresan como [28]: 


$$
\chi_{G}=\left\{\begin{array}{l}
\frac{1}{\varepsilon_{\mathrm{GaP}}(P)}+\frac{2 \pi R^{2}}{a^{2} \sqrt{3}}\left(1-\frac{1}{\varepsilon_{\mathrm{GaP}}(P)}\right) ; \text { para } \vec{G}=0 \\
\frac{4 \pi R}{a^{2} \sqrt{3} G}\left(1-\frac{1}{\varepsilon_{\mathrm{GaP}}(P)}\right) J_{1}(G R) ; \text { para } \vec{G} \neq 0
\end{array}\right.
$$

donde $J_{1}(G R)$ es la función de Bessel de primer orden.

\section{Resultados}

En la Tab.1 se muestra los valores de la permitividad dieléctrica del GaP para diferentes presiones hidrostáticas de acuerdo con la Ec.(9), utilizadas para estudiar y analizar la EBF.

Tabla 1. Valores de la permitividad dieléctrica para una presión hidrostática de $\mathrm{P}=0 \mathrm{kbar}, \mathrm{P}=40 \mathrm{kbar}, \mathrm{P}=80 \mathrm{kbar}$ y $\mathrm{P}=100 \mathrm{kbar}$.

\begin{tabular}{cc}
\hline $\mathrm{P}(\mathrm{kbr})$ & $\varepsilon_{\mathrm{GaP}}$ \\
\hline 0 & 10.87425 \\
40 & 10.46041 \\
80 & 10.0623 \\
100 & 9.8689 \\
\hline
\end{tabular}

A continuación, se presentan algunas figuras que muestran los resultados de esta investigación usando el método de expansión en ondas planas, desarrollado en un lenguaje de programación con base en la ejecución realizada por G. Shangping [29], implementando el sistema de cómputo de MATLAB.

La Fig.3 muestra la EBF del CF-2D regular para la polarización TM, para una presión hidrostática de $P=$ 0kbar, diagonalización la Ec.(8) con una base de 41 ondas planas para cada dirección de simetría en el contorno de la zona reducida de Brillouin, cuyos vértices son $\Gamma:\left(k_{x}=\right.$ $\left.0 ; k_{y}=0\right), \quad M:\left(k_{x}=0 ; k_{y}=\frac{2 \pi}{\sqrt{3} a}\right), \quad \mathrm{K}:\left(k_{x}=\frac{2 \pi}{3 a} ; k_{y}=\right.$ $\left.\frac{2 \pi}{\sqrt{3} a}\right)$.

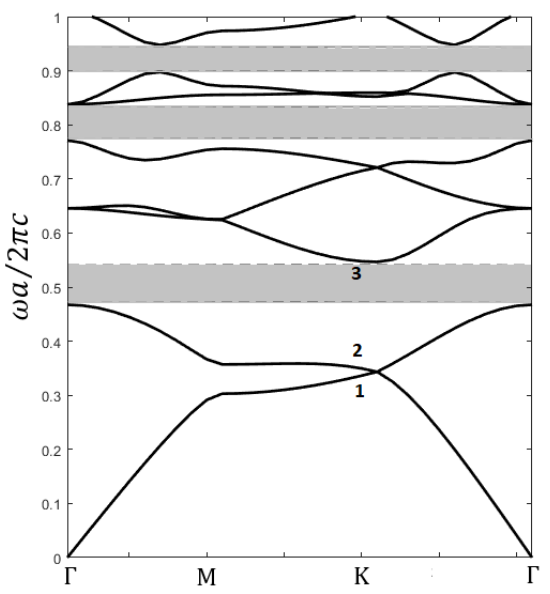

Figura 3. Estructura de banda fotónica del CF-2D con geometría hexagonal compuesto por agujeros de aire insertados en $\mathrm{GaP}$, para polarización TM a una presión hidrostática de $\mathrm{P}=0 \mathrm{kbar}$.

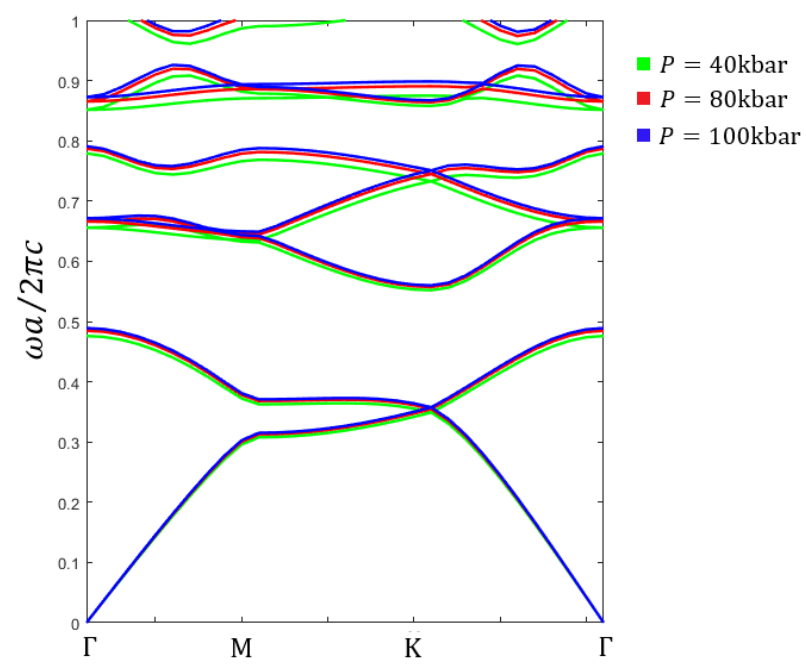

Figura 4. Estructura de banda fotónica del CF-2D con geometría hexagonal compuesto por agujeros de aire insertados en $\mathrm{GaP}$, para polarización TM a una presión hidrostática de $\mathrm{P}=40 \mathrm{kbar}$ (línea verde), $\mathrm{P}=80 \mathrm{kbar}$ (línea roja) y P $=100 \mathrm{kbar}$ (línea azul).

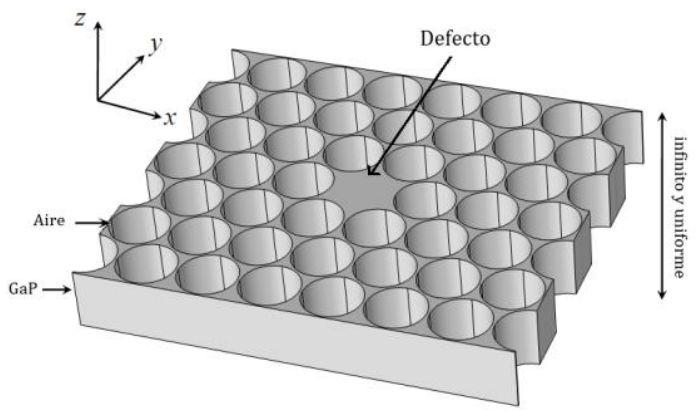

Figura 5. Esquema del CF-2D de red hexagonal donde se ha eliminado un agujero de aire dentro del $\mathrm{GaP}$ de radio $\mathrm{R}=0.48 a$.

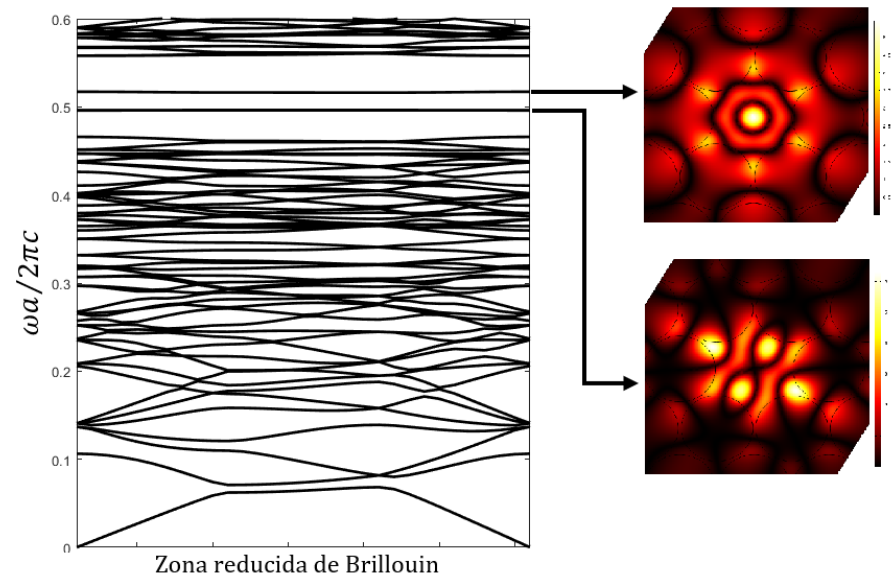

Figura 6. Estructura de banda fotónica del CF-2D defectivo con geometría hexagonal, para polarización TM a una presión hidrostática de $\mathrm{P}=0 \mathrm{kbar}$ y perfil de intensidad del campo eléctrico de los modos confinados. 


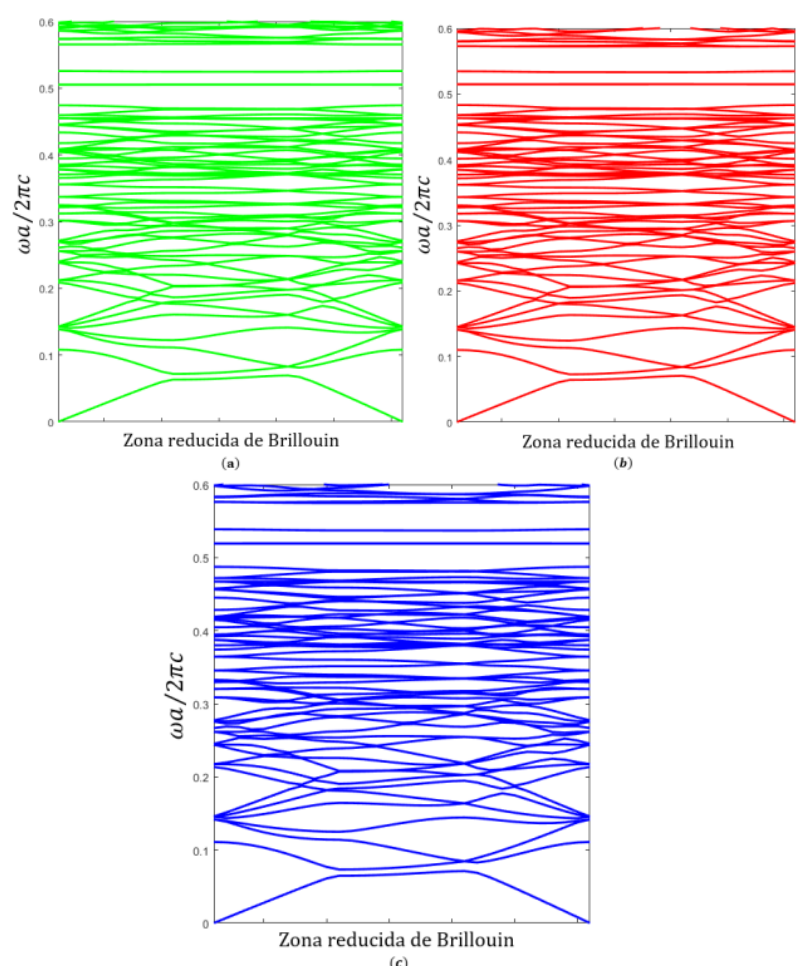

Figura 7. Estructura de banda fotónica del CF-2D defectivo, para polarización TM a una presión hidrostática de (a) $\mathrm{P}=40 \mathrm{kbar}$, (b) $\mathrm{P}=$ $80 \mathrm{kbar}$, (c) $\mathrm{P}=100 \mathrm{kbar}$.

\section{Discusiones}

Esta investigación se centra en estudiar el comportamiento de la EBF de un cristal fotónico bidimensional (CF-2D) con geometría hexagonal, compuesto por agujeros de aire de radio $R=0.48 a$ insertados en el material de GaP como se muestra en la Fig.1.

De igual forma se puede apreciar la existencia más notoria de una BFP entre la segunda y tercera banda, debido a la amplia diferencia de frecuencias correspondiente entre los estados consecutivos, producto de la interferencia destructiva de las ondas electromagnéticas dentro del CF. En la Fig.4 se presenta la EBF cuando aplicamos una presión hidrostática de $P=40 \mathrm{kbar}, P=80 \mathrm{kbar}$ y $P=100 \mathrm{kbar}$, obteniendo un desplazamiento de la EBF a regiones de alta frecuencia debido a que la función dieléctrica disminuye a medida que se aumenta la presión hidrostática sin modificar la estructura del cristal.

Para producir estados localizados con energías que se encuentran dentro de la BFP, se introduce artificialmente una impureza o un defecto puntual en el CF que rompe la periodicidad del sistema. Se estudia la EBF del CF-2D defectivo al eliminar un agujero de aire en la estructura, como se muestra en la Fig.5 utilizando el MEOP y la técnica de la supercelda [30]. La construcción repetitiva de la supercelda se realiza a lo largo de las diferentes direcciones espaciales para formar nuevamente una estructura periódica, garantizando que la separación de los defectos consecutivos sea la adecuada para que exista las mismas propiedades espectrales que el cristal original, sin producir solapamiento entre los defectos para obtener los valores correctos de las frecuencias de los modos confinados. Se consideró una supercelda de tamaño $5 a \times 5 a$. En la Fig. 6 se representa la EBF del CF-2D defectivo, se observó la aparición de un mayor número de bandas, debido a que la primera zona de Brillouin en la supercelda es más pequeña que en la celda unitaria primitiva, sin embargo, el ancho y la posición del PBG permanecen iguales. Se localizaron modos confinados o defectivos dentro del BFP, con frecuencias $\omega_{1}=$ (0.496) $2 \pi c / a$ y $\omega_{2}=(0517) 2 \pi c / a$, con energías contantes para cualquier vector $\vec{k}$ y sus velocidades de grupo iguales a cero; utilizando la Ec.(6) se calculó los perfiles de intensidad del campo eléctrico asociados a los modos defectivos, en realidad son modos degenerados, debido a que, en este tipo de estructuras no existe una sola banda defectiva sino un desdoblamiento de bandas muy cercanas que reflejan un degeneramiento. En la Fig.7 se muestra la EBF para diferentes valores de la presión hidrostática, se encontró un desplazamiento de los modos defectivos a frecuencias más altas, por lo que las energías asociadas a estos modos localizados son mayores.

\section{Conclusiones}

Según los resultados mostrados anteriormente bajo los efectos de la presión hidrostática en la EBF en un CF-2D con red hexagonal compuesto por agujeros de aire dentro de un material semiconductor $(\mathrm{GaP})$ para la polarización TM se calculó numéricamente la ecuación maestra para determinar la EBF para el CF regular; se encontraron regiones de BFP producto de las interferencias destructivas de los modos electromagnéticos. Al aumentar la presión hidrostática se presentó un desplazamiento de la estructura de bandas a regiones de alta frecuencia debido a la disminución de la permitividad dieléctrica del GaP. El resultado de introducir un defecto en el cristal fotónico eliminando un agujero de aire, genera modos defectivos con energías bien definidas dentro de la BFP, conocidos como modos confinados, además, el ancho y la posición del BFP no presentaron cambios con esta modificación. Sin embargo, el aumento de la presión hidrostática en la permitividad dieléctrica del GaP origina un desplazamiento de los modos confinados a valores de altas frecuencias y el ancho de la BFP disminuye. En consecuencia, este análisis genera un aporte al estudio de materiales semiconductores alternos como al $\mathrm{SiO}_{2}$ o al GaAs para el desarrollo de dispositivos optoelectrónicos sometidos a una perturbación externa como la presión.

\section{Reconocimientos}

Los autores agradecen a la Vicerrectoría de Investigación y Proyección Social (VIPS) de la Universidad Surcolombiana por el apoyo financiero al proyecto titulado "Cálculo de la distribución del modo fundamental en fibra microestructurada por el método de expansión en ondas 
planas" con código 3308 dentro de la convocatoria interna $\mathrm{N}^{\circ}$ PTG01, el cual hace parte de esta contribución.

\section{Referencias}

[1] Massaro A., Photonic Crystals: Introduction, Applications and Theory, 1st Edition, InTechOpen, 2012.

[2] Joannopoulos J., Johnson S., Winn J., Meade R., Photonic Crystals: Molding the Flow of Light, 2nd Edition, Princeton University Press, 2008.

[3] Yablonovitch E., Inhibited Spontaneous Emission in Solid-State Physics and Electronics. Phys. Rev. Lett. 58(20) (1987) 2059-2062. https://doi.org/10.1103/PhysRevLett.58.2059

[4] S Jhon., Strong localization of photons in certain disordered dielectric superlattices. Phys. Rev. Lett. 58(23) (1987) 2486-2489. https://doi.org/10.1103/PhysRevLett.58.2486

[5] Dressel M., Grüner G. Electrodynamics of Solids: Optical Properties of Electrons in Matter, 1st Edition, Cambridge University Press, 2002.

[6] Aschcroft N., Mermin D., Wei D., Solid State Physics, Cengage Learning Asia, 2016.

[7] Gong Q., Hu X., Photonic crystals: Principles and Applications, 1st Edition, Pan Stanford Publishing, 2013.

[8] Obayya, S., Farhat M., Hameed O., Areed N., Computational Liquid Crystal Photonics: Fundamentals, Modelling and Application, Wiley, 2016.

[9] Hameed M., Obayya S., Computational Photonic Sensors, Springer, 2018.

[10] Yablonovitch E., Photonic crystals: semiconductors of light. Sci. Am. 285(6) (2001) 46-55. https://doi.org/10.1038/scientificamerican1201-46

[11] Sanchez A., Porta A., Orozco S., Photonic band-gap and defect modes of a one-dimensional photonic crystal under localized compression. J. Appl. Phys 121(17) (2017),173101. https://doi.org/10.1063/1.4982760

[12] Gharaati A., Zare Z., The effect of temperature on one-dimensional nanometallic photonic crystals with coupled defects. Pramana-J. Phys. 88(5) (2017) 75.

https://doi.org/10.1007/s12043-017-1380-5

[13] Berman O., Lozovik Y., Eiderman S., Coalson R., Superconducting photonic crystals: numerical calculations of the band structure. Phys. Rev. B 74(9) (2006) 092505. https://doi.org/10.1103/PhysRevB.74.092505

[14] Liu N., Guo H., Fu L., Kaiser S., Schweizer H., Giessen H., Threedimensional photonic metamaterials at optical frequencies. Nat. Mater 7(1) (2008) 31-37. https://doi.org/10.1038/nmat2072

[15] Lin M., Jao R., Finite element analysis of photon density of states for two-dimensional photonic crystals with in-plane light propagation. Opt. Express 15(1) (2007) 207-218. https://doi.org/10.1364/OE.15.000207

[16] Taflove A., Hagness S., Computational Electrodynamics: The FiniteDifference Time-Domain Method, 3rd Edition, Artech House Publishers, 2005.
[17] Shumpert J. Modeling of Periodic Dielectric Structures (Electromagnetic Crystals), Ph.D. thesis, University of Michigan, 2001.

[18] Skorobogatiy M., Yang J., Fundamentals of Photonic Crystal Guiding, 1st Edition, Cambridge University Press, 2009.

[19] Sakoda K., Optical Properties of Photonic Crystals, Springer, 2nd Edition, 2004.

[20] Sözüer H., Haus J., Inguva R., Photonic bands: convergence problems with the plane-wave method. Phys. Rev. B 45(24) (1992) $13962-$ 13972.

https://doi.org/10.1103/PhysRevB.45.13962

[21] Aly A., Elsayed H., El-Naggar S., Tuning the flow of light in twodimensional metallic photonic crystals based on Faraday Effect. J. Mod. Optics 64(1) (2017) 74-80. https://doi.org/10.1080/09500340.2016.1208298

[22] Duque C., Mora-Ramos M., The two-dimensional square and triangular photonic lattice under the effects of magnetic field, hydrostatic pressure and temperature. Opt. Quant. Electron 44(8-9) (2012) 375-392. https://doi.org/10.1007/s11082-012-9545-4

[23] Porras-Montenegro N., Duque C., Temperature and hydrostatic pressure effects on the photonic band structure of a $2 \mathrm{D}$ honeycomb lattice. Phys. E 42(6) 2010 1865-1869. https://doi.org/10.1016/j.physe.2010.02.016

[24] Segovia, F., Vinck H., Effects of hydrostatic pressure on the band structure in two-dimensional semiconductor square photonic lattice with defect. Phys. B 545 (2018) 203-209. https://doi.org/10.1016/j.physb.2018.06.014

[25] Orfanidis S., Electromagnetic Waves and Antennas, NJ Rutgers University, New Brunswick, 2002.

[26] Kittel C., Introduction to Solid State Physics, 8th Edition, Wiley, 2004.

[27] Samara G., Temperature and pressure dependences of the dielectric constants of semiconductors. Phys. Rev. B 27(6) (1983) 3494-3505. https://doi.org/10.1103/PhysRevB.27.3494

[28] Sukhoivanov I., Guryev I., Photonic Crystals: Physics and Practical Modeling, Springer, 2009.

[29] Shangping., G., Photonic Crystals: Modeling and Simulation, Ph.D. thesis, Department of Electrical \& Computer Engineering, Old Dominion University, Norfolk VA, 2003.

[30] Wu L., Zhuang F., He S., Degenerancy analysis for a supercell of a photonic crystal and its applications to the creation of band gaps. Phys. Rev. E 67 (2 Pt 2) (2003) 026612. https://doi.org/10.1103/PhysRevE.67.026612 